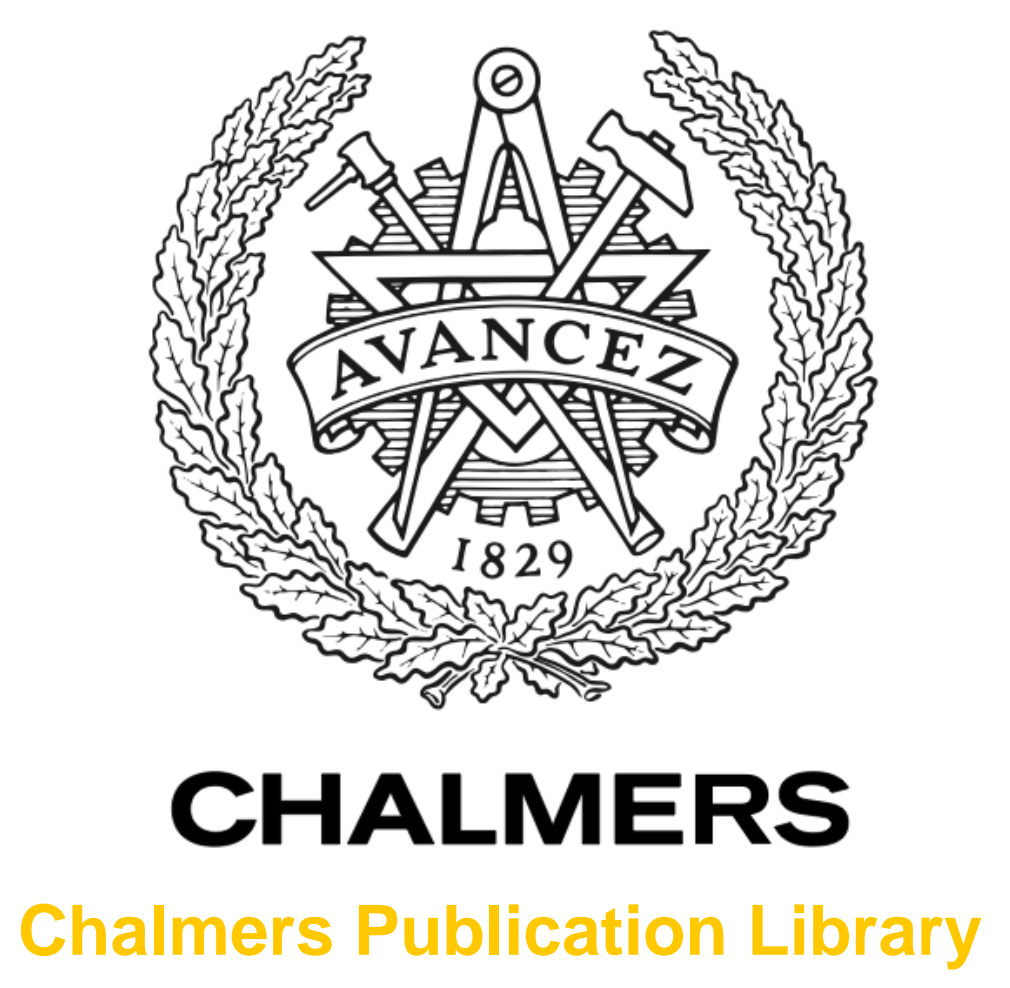

Reachability Analysis of Cooperative Adaptive Cruise Controller

This document has been downloaded from Chalmers Publication Library (CPL). It is the author's version of a work that was accepted for publication in:

2012 15th International IEEE Conference on Intelligent Transportation Systems, ITSC 2012, Anchorage, AK, 16 - 19 September 2012 (ISSN: 2153-0009)

Citation for the published paper:

Kianfar, R. ; Falcone, P. ; Fredriksson, J. (2012) "Reachability Analysis of Cooperative Adaptive Cruise Controller". 2012 15th International IEEE Conference on Intelligent

Transportation Systems, ITSC 2012, Anchorage, AK, 16 - 19 September 2012 pp. 1537 1542.

http://dx.doi.org/10.1109/ITSC.2012.6338839

Downloaded from: http://publications.lib.chalmers.se/publication/165162

Notice: Changes introduced as a result of publishing processes such as copy-editing and formatting may not be reflected in this document. For a definitive version of this work, please refer to the published source. Please note that access to the published version might require a subscription. 


\title{
Reachability Analysis of Cooperative Adaptive Cruise Controller
}

\author{
Roozbeh Kianfar, Paolo Falcone, Jonas Fredriksson
}

\begin{abstract}
In this paper, a set based approach to safety analysis of Adaptive Cruise Control (ACC) and Cooperative Adaptive Cruise Control (CACC) is presented. Reachability analysis techniques are used to compare the minimum safe intervehicle distances which can be achieved with ACC and CACC controllers. Not surprisingly, the results indicate that a shorter inter-vehicle distance can be achieved with a CACC controller. The presented method can also be used to design the required inter-vehicle distance for a given controller. Furthermore, we show how backward reachability analysis and invariant set theory can be used to find the Maximal Asymptotic Safe Set. This is defined as a set of position error, relative speeds and acceleration, which a given controller is guaranteed to control to the desired speed and inter-vehicle distance, while fulfilling vehicle physical constraints and avoiding rear-end collisions with the preceding vehicle. The calculation of the Maximal Asymptotic Safe Set is demonstrated for ACC and CACC controller designed based on mixed $H_{2} / \infty$ state feedback. Finally, the calculation of the Maximal Asymptotic Safe Set is extended to the case of vehicle model uncertainties.
\end{abstract}

\section{INTRODUCTION}

Road congestions are common ground in transportation systems, which can result in increased emissions and fuel consumption, accident risk and traveling time. Intelligent transportation systems can mitigate congestion problems, see [1]. Platooning is an example of intelligent transportation systems. Controlling the inter-vehicle distances while avoiding rear-end collisions in a train of vehicles is the idea underlying vehicles platoon control. The main outcome of platooning is reduced inter-vehicle distance between vehicles, solely increased traffic throughput and better usage of roads capacity. The idea of platooning can be traced back to the eighties when California's Partners for Advanced Transit and Highways (PATH) program was established to study and develop vehicle-highway cooperation and communication systems, [2] and [3]. Platooning has been furthered investigated by many researchers, e.g., [4] and [5].

Nowadays, adaptive cruise control (ACC) and Cooperative adaptive cruise control (CACC) can be employed as means of enabling platooning, [6]. Adaptive cruise control (ACC) is a functionality which can be found in many modern vehicles. ACC is used to automatically adjust the vehicle's velocity with respect to the preceding vehicle. Cooperative adaptive cruise control (CACC) is an enhanced version of ACC, which exploits communicated information from the preceding vehicle. Results of studies in [1] and [7] indicate the potential of $\mathrm{CACC}$ in improving the traffic flow and reducing congestion.

Roozbeh Kianfar, Paolo Falcone and Jonas Fredriksson are with Department of Signals and Systems, Mechatronics, Chalmers University of Technology, 41296 SE, Göteborg, Sweden roozbeh, falcone, jonas. fredriksson@chalmers.se

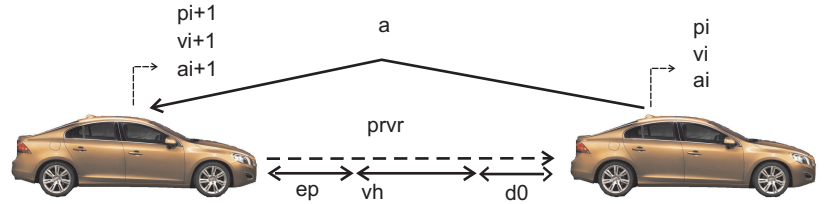

Fig. 1. Two adjacent vehicles in the platoon.

Recently, CACC, as a practical approach to improve the traffic flow, has attracted the attention of many researchers [8] and [9]. In [9], it is shown that, using CACC makes it possible to reduce the inter-vehicle distance compared to ACC. In [10], the minimum required headway time to preserve string stability in a homogeneous platoon equipped with local CACC controllers is derived. It is of interest to investigate how much the inter-vehicle distance can be reduced without jeopardizing safety using CACC rather than an ACC. In [4], it is shown that the quantitative aspect of this analysis depends on the specific structure of the controller.

In this work, the aforementioned results are extended to the safety verification of a platoon equipped with CACC. Hence, reachability techniques and invariant set theory are employed as a tool for safety analysis of vehicles equipped with ACC and CACC. Forward reachability analysis technique is used to calculate the minimum required safe distance between vehicles to avoid collisions. As an example, the proposed technique is applied to an ACC and a CACC controller. Furthermore, a set of constraints on the vehicle states are introduces, which represent the safe driving mode. An algorithm based on backward reachability analysis and invariant set theory is provided to compute the maximal asymptotic safe set, that is, the set of initial states which guarantees constraints satisfaction over future time. The algorithm is also extended to handle parametric uncertainty in the vehicle model. As an example the algorithm is applied to a CACC with uncertainty in the vehicle model.

\section{VEHICLE MODELING}

Consider two adjacent vehicles, as shown in Fig. 1. Let $p_{i}, v_{i}$ and $a_{i}$ denote the position, velocity and acceleration of the preceding vehicle and $p_{i+1}, v_{i+1}$ and $a_{i+1}$ denote the position, velocity and acceleration of the following vehicle (the ego vehicle), respectively. Denote by $e_{p}$ the position error w.r.t. a desired distance from the preceding vehicle, i.e., $e_{p}=p_{i}-p_{i+1}-d_{0}-v_{i+1} h_{i+1}$, where $d_{0}$ and $h_{i+1}$ are a constant safety distance and the constant headway time, respectively. The headway time is the time that the ego vehicle takes to reach the preceding vehicle while traveling 
at its current speed. The error dynamics are then described by the following set of equations

$$
\begin{aligned}
& \dot{e}_{p}=e_{v}-a_{i+1} h_{i+1}, \\
& \dot{e}_{v}=a_{i}-a_{i+1} .
\end{aligned}
$$

where $e_{v}$ is the relative velocity. The acceleration of the ego vehicle, $a_{i+1}$ is assumed to be described by a first order system,

$$
a_{i+1}=\frac{K_{i+1}}{\tau_{i+1} s+1} e^{-\theta_{i+1} s} a_{i+1}^{\mathrm{des}},
$$

where $K_{i+1}, \tau_{i+1}$ and $\theta_{i+1}$ are the steady state gain, the time constant of the actuator (engine and brake) and the actuator delay, respectively and $a_{i+1}^{\text {des }}$ is the demanded acceleration, [11]. The model (1)-(2) can then be written in a state-space form as

$$
\dot{x}(t)=A x(t)+B_{u} u(t-\theta)+B_{\omega} \omega(t),
$$

where

$$
\begin{aligned}
A & =\left[\begin{array}{ccc}
0 & 1 & -h_{i+1} \\
0 & 0 & -1 \\
0 & 0 & -1 / \tau_{i+1}
\end{array}\right], \\
B_{u} & =\left[\begin{array}{c}
0 \\
0 \\
\frac{K_{i+1}}{\tau_{i+1}}
\end{array}\right], B_{\omega}=\left[\begin{array}{l}
0 \\
1 \\
0
\end{array}\right],
\end{aligned}
$$

and

$$
\begin{aligned}
x & =\left[\begin{array}{lll}
e_{p} & e_{v} & a_{i+1}
\end{array}\right]^{\mathrm{T}} \\
u & =a_{i+1}^{\mathrm{des}} \\
\omega & =a_{i}
\end{aligned}
$$

are the state, the control and the disturbance vectors, respectively. Notice that the acceleration of the preceding vehicle is considered as a disturbance.

\section{CONTROL DESIGN}

Primary objective of autonomous systems, i.e., ACC and CACC controllers is to provide comfort for the drivers by maintaing the desired speed and position. However, such systems can also improve safety by avoiding collision. In this work, the focus is mainly on safety analysis of ACC and CACC controllers. The safety analysis is demonstrated on a $H_{2 / \infty}$ state feedback controller, which is designed using Linear Matrix Inequality (LMI), see [12]. Controller design problem is formulated as an optimization problem where a weighted sum of the two objective functions is minimized by the controller depicted in Fig. 2.

$$
\begin{array}{r}
\min _{u} \alpha\|F\|_{\infty}+\beta\|H\|_{2} \\
\text { s.t }\|F\|_{\infty} \leq \gamma \\
\|H\|_{2} \leq \eta
\end{array}
$$

where $u$ is the control input and $H$ and $F$ are transfer functions from the disturbance $\omega$ to the desired outputs,

$$
z_{2}=\left[\begin{array}{ll}
e_{p} & u
\end{array}\right]^{\mathrm{T}}, z_{\infty}=\left[\begin{array}{ll}
a_{i+1} & e_{p}
\end{array}\right]^{\mathrm{T}},
$$

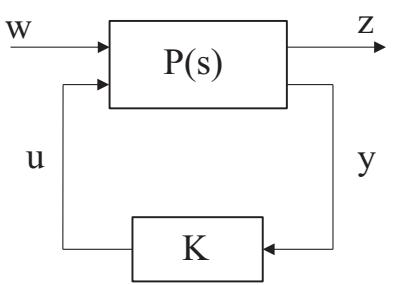

Fig. 2. closed loop system with $P$ the open loop dynamic and $K$ the controller

respectively. $z_{2}$ and $z_{\infty}$ can be written in a state space form as follows,

$$
\begin{aligned}
z_{2}(t) & =C_{2} x(t)+D_{2,1} \omega(t)+D_{2,2} u(t) \\
z_{\infty}(t) & =C_{\infty} x(t)+D_{\infty, 1} \omega(t)+D_{\infty, 2} u(t),
\end{aligned}
$$

In this paper, $u$ is calculated for two cases where $i$ ) only the state measurement is available and the disturbance $\omega$ is unknown (feedback control, ACC) ii) where the disturbance $\omega$ is also measured (feedback/feedforward, CACC).

\section{A. ACC and CACC controllers}

The ACC controller as mentioned earlier is a state feedback controller $u=K x(t)$. Under this control law the closed loop dynamic can be written as:

$$
\begin{aligned}
\dot{x}(t) & =\left(A+B_{u} K\right) x(t)+B_{\omega} \omega(t), \\
z_{\infty}(t) & =\left(C_{\infty}+D_{\infty, 2} K\right) x(t)+D_{\infty, 1} \omega(t), \\
z_{2}(t) & =\left(C_{2}+D_{2,2} K\right) x(t)+D_{2,1} \omega(t),
\end{aligned}
$$

where $x, u$ and $\omega$ are the state, control signal and disturbance, respectively. In this case, the acceleration of the preceding vehicle $\omega=a_{i}$ is treated as an unknown but bounded disturbance.

To design the CACC controller, the disturbance $\omega=a_{i}$ is assumed to be measured. The feedback/feedforward control law is represented as $u=K x(t)+K_{f} \omega(t)$, where $K$ and $K_{f}$ are the feedback and feedforward gain, respectively. The closed loop system then becomes,

$$
\begin{aligned}
\dot{x}(t) & =\left(A+B_{u} K\right) x(t)+\left(B_{u} K_{f}+B_{\omega}\right) \omega(t), \\
z_{\infty}(t) & =\left(C_{\infty}+D_{\infty, 2} K\right) x(t)+\left(D_{\infty, 1}+D_{\infty, 2} K_{f}\right) \omega(t), \\
z_{2}(t) & =\left(C_{2}+D_{2,2} K\right) x(t)+D_{2,1} \omega(t),
\end{aligned}
$$

The closed loop transfer function from $\omega \rightarrow z_{\infty}$, can be written as,

$$
H(s)=C_{H}\left(S I-A_{H}\right)^{-1} B_{H}+D_{H},
$$

where $A_{H}=A+B_{u} K, B_{H}=B_{u} K_{f}+B_{\omega}, C_{H}=C_{\infty}+$ $D_{\infty, 2} K$ and $D_{H}=D_{\infty, 1}+D_{\infty, 2} K_{f}$. Equation (14), shows that, the feedforward gain $K_{f}$ adds an additional degree of freedom to the optimization problem (9).

It should be noted that, the advantages of designing the controller using the described approach for the analysis is, since the controller design is formulated as an optimization problem, both controllers, i.e., the ACC controller and CACC controller are the optimal controllers according to (9). Therefore, issues like, controller tuning and controller design criterion are not a hinder in the analysis. 


\section{Reachability analysis of Platoon}

To guarantee safety, it is necessary to guarantee that error trajectories do not violate certain constraints. In this work, reachability analysis technique is employed to $i$ ) investigate the effect of additional information to the controller, i.e., acceleration of preceding vehicle provided by $\mathrm{V} 2 \mathrm{~V}$ communication, on the inter-vehicle distance. This means, a comparison between the introduced position error $e_{p}$ for an ACC and a CACC ii) finding the largest set of initial state, which guarantees persistent feasibility. This will be defined formally next.

\section{A. Background and preliminaries}

In this section, a few definitions are introduced and basic results on reachability analysis, which are used in the rest of the paper, are presented. For further information regarding invariant set theory and reachability analysis, we refer to [13] and [14].

Definition 1 A polyhedron $\mathcal{P} \in \mathbb{R}^{n}$ is the intersection of finite number of closed halfspaces in $\mathbb{R}^{n}$

$$
\mathcal{P}=\left\{x \in \mathbb{R}^{n} \mid H x \leq h\right\}
$$

Remark: A closed polyhedron is called a polytope.

Definition 2 The Minkowski sum of two polytopes $\mathcal{R}$ and $\mathcal{Q}$ is a polytope defines as,

$$
\mathcal{R} \oplus \mathcal{Q}=\left\{x+y \in \mathbb{R}^{n} \mid x \in \mathcal{R}, y \in \mathcal{Q}\right\}
$$

Definition 3 The Pontryagin difference of two polytopes $\mathcal{R}$ and $\mathcal{Q}$ is a polytope defines as,

$$
\mathcal{R} \ominus \mathcal{Q}=\left\{x \in \mathbb{R}^{n} \mid x+q \in \mathcal{R}, \forall q \in \mathcal{Q}\right\}
$$

Definition 4 Composition of an affine mapping $f$ and a polyhedron $\mathcal{P}$, with $f$ as,

$$
f: z \in \mathbb{R}^{m} \mapsto A z+b, \quad A \in \mathbb{R}^{m_{A} \times m}, \quad b \in \mathbb{R}^{m}
$$

is defined as,

$$
f \circ \mathcal{P}=\left\{y \in \mathbb{R}^{m} \mid y=A x+b \quad \forall x \in \mathbb{R}^{n}, \quad H x \leq h\right\}
$$

Denote by $f_{a}$ the state update function of an autonomous system,

$$
x(k+1)=f_{a}(x(k), \omega(k)),
$$

where $x(k)$ and $\omega(k)$ are the state and disturbance vector, respectively. The system (20) is subject to the following constraint,

$$
x \in \mathcal{X}, \omega \in \mathcal{W},
$$

where $\mathcal{X}$ and $\mathcal{W}$ are polytopes in $\mathbb{R}^{n}$ and $\mathbb{R}^{d}$, respectively.

Definition 5 For the autonomous system (20), we denote the robust one-step reachable set for initial states $x(0)$ contained in the set $\mathcal{S}$ as,

$\operatorname{Reach}^{f_{a}}(\mathcal{S}, \mathcal{W})=\left\{x \in \mathbb{R}^{n}: \exists x(0) \in \mathcal{S}, \exists \omega \in \mathcal{W} \mid x=f_{a}(x(0), \omega)\right\}$

Definition 6 For the autonomous system (20), the robust Pre set is defined as the dual of one-step reachable set,

$\operatorname{Pre}^{f_{a}}(\mathcal{T}, \mathcal{W})=\left\{x \in \mathbb{R}^{n}: f_{a}(x(k), \omega(k)) \in \mathcal{T}, \forall \omega \in \mathcal{W}\right\}$, where $\mathcal{T}$ is the target set.

In the next section, using reachability analysis technique, the effect of disturbance on the dynamic states, i.e., position error, velocity error and acceleration is investigated. The study comprises previously introduced ACC and CACC controllers.

\section{B. Reachable set for ACC and CACC controllers}

The closed loop systems (12) and (13) are discretized with sampling time $T_{s}$. The discretized autonomous systems are represented for ACC and CACC controller, respectively.

$$
\begin{gathered}
x(k+1)=A_{d} x(k)+E_{d} \omega(k), \\
x(k+1)=\tilde{A}_{d} x(k)+\tilde{E}_{d} \omega(k)
\end{gathered}
$$

The robust forward reachable set of the closed loop systems (22) and (23) can be computed as,

$$
\begin{gathered}
\operatorname{Reach}^{\left(A_{d}, E_{d}\right)}(\mathcal{S}, \mathcal{W})=\left(A_{d} \circ \mathcal{S}\right) \oplus\left(E_{d} \circ \mathcal{W}\right), \\
\operatorname{Reach}^{\left(\tilde{A}_{d}, \tilde{E}_{d}\right)}(\mathcal{S}, \mathcal{W})=\left(\tilde{A}_{d} \circ \mathcal{S}\right) \oplus\left(\tilde{E}_{d} \circ \mathcal{W}\right)
\end{gathered}
$$

Next we show how reachability analysis methods can be used to study the minimum "safe" distance in case of emergency braking of the preceding vehicle. Assume the maximum deceleration of the preceding vehicle is $\omega=a_{i}^{\max }=-6 \frac{\mathrm{m}}{\mathrm{s}^{2}}$. Hence, a vehicle traveling with velocity $v=21 \frac{m}{s}$ needs $t=$ $3.5 s$ to stop. However, we should note that the deceleration $a_{i}^{\max }=-6 \frac{m}{s^{2}}$ is considered as the maximum deceleration to ease our computation, though it does not cover the whole range of emergency braking.

In Fig. 3(a) and Fig. 3(b), the projections of reachable sets on $\left(e_{p}, e_{v}\right)$ plane from an initial set $\mathcal{S}$ around origin, i.e., equilibrium point of platoon, is depicted. The initial set $\mathcal{S}$ represents the set of states steady state operation of the two vehicles, i.e., small position and velocity errors. The reachable set are calculated for the closed loop systems with the ACC (22) and the CACC controller (23). The closed loop systems are driven by the disturbance $\omega \in[-6,-1]$. The headway time $h_{i+1}$ is set to zero for this analysis. Note that, the reachable sets are computed for 35 steps with the sampling time $T_{s}=0.1 s$. The reachable sets could be calculated over longer time horizons. However, since Minkowski sum and projection are computationally expensive operations, for the sake of simplicity only used 35 steps are used. The reachable sets are calculated by using the Mutiparametric toolbox for Matlab, [15].

Fig. 3, shows that the CACC controller introduces smaller position and velocity errors compared to the ACC controller. Fig. 3(a) indicates that, for an ACC controller, at least a safety distance of $d_{0, \min }=3.4 \mathrm{~m}$ is required to avoid collision in emergency braking. While, for a CACC controller, a minimum safety distance $d_{0, \min }=1.5 \mathrm{~m}$ is enough to ensure that collision does not occur under the considered conditions. In Fig. 4, the projection of reachable sets on the plane $\left(a, e_{p}\right)$ are shown. Fig. 4 indicates that, the ACC controller commanded slightly larger acceleration which may saturate the actuators earlier compared to the CACC controller. 


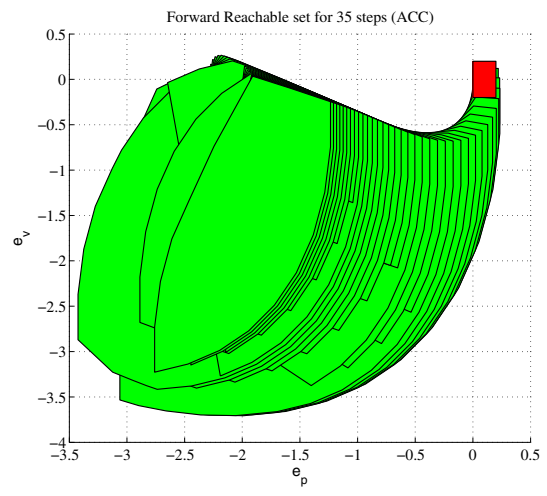

(a) Forward reachable set for ACC

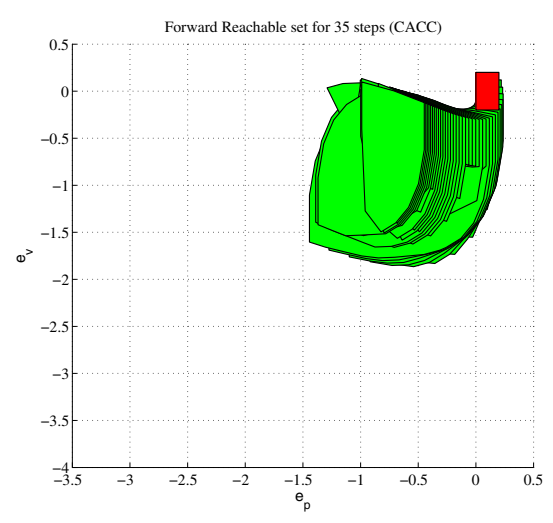

(b) Forward reachable set for CACC

Fig. 3. Projection of forward reachable set for 35 steps and the set of initial state, in case of emergency braking $\omega \in(-6,-1)$ are shown in green and red, respectively. $h=0$ (headway)

\section{Maximal asymptotic safe set}

In this section, backward reachability analysis is employed to find a safe set which guarantees safety while satisfying vehicle's actuators limitations in presence of a bounded disturbance. Hence, a safe region on the state space is introduced where platoon safety is guaranteed for any state belonging to this region. To guarantee safety, maintaining performance and fulfilling actuators limitations, following constraints must be fulfilled by the ego vehicle.

1) Safety: Safety requirements imply that a safe minimum distance should be maintained from the preceding vehicle in order to reduce the risk of collisions. Hence, the safety requirements on the inter-vehicle spacing can be rewritten as

$$
e_{p, \min } \leq e_{p}(k) \leq e_{p, \max }, \quad \forall k \geq 0,
$$

where $e_{p, \min }$ and $e_{p, \max }$ are the minimum and maximum allowed distance error, respectively. We observe that, while $e_{p, \max }$ can be selected according to performance criteria, the lower bound in (26) forces the distance between the ego and the preceding vehicle to be higher than $d_{0}+v_{i+1} h_{i+1}$.

2) Performance: Since the primary objective of the cooperative driving system is to regulate the vehicle velocity to the platoon velocity, the relative speed between the two

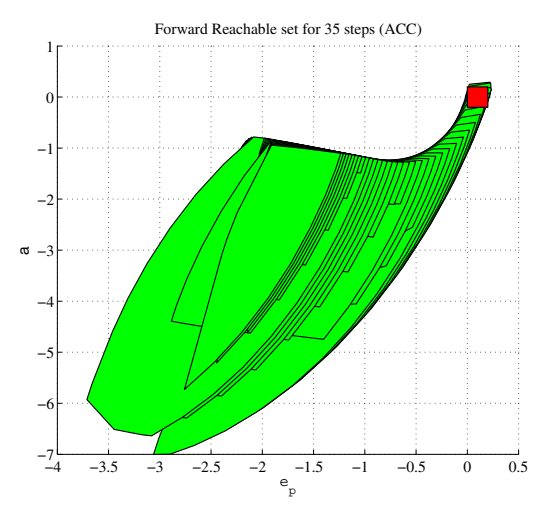

(a) Forward reachable set for ACC, in case of emergency braking $\omega \in(-6,-1)$

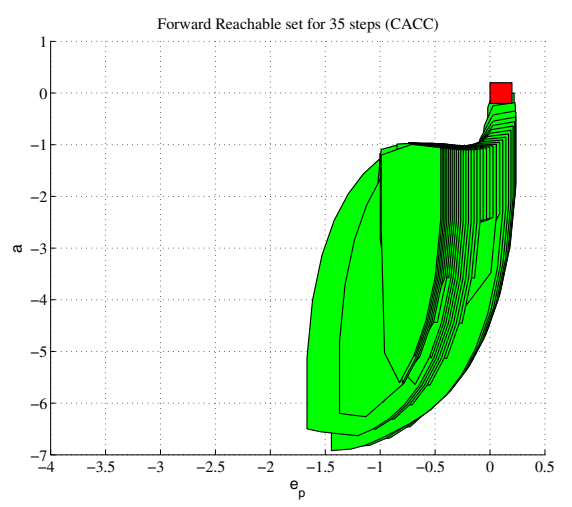

(b) Forward reachable set for CACC, in case of emergency braking $\omega \in(-6,-1)$

Fig. 4. Projection of forward reachable set for 35 steps and the set of initial state are shown in green and red, respectively. $h=0$ (headway)

adjacent vehicles is constrained, i.e.,

$$
e_{v, \min } \leq e_{v}(k) \leq e_{v, \max }, \quad \forall k \geq 0,
$$

where $e_{v, \min }$ and $e_{v, \max }$ are the minimum and maximum allowed deviation from the preceding vehicle's velocity, respectively.

3) Actuator limitations: Due to actuator limitation (the controlled engine and brake), the amount of acceleration/deceleration that the vehicle can deliver is also limited. Hence, to comply with the actuator limits, the acceleration of the vehicle is constrained as following,

$$
a_{\min } \leq a(k) \leq a_{\max }, \quad \forall k \geq 0,
$$

where $a_{\min }$ and $a_{\max }$, are the minimum and maximum acceleration that the actuator can deliver, respectively. The constraints (26), (27) and (28) can be written as a polytope,

$$
H_{x} x \leq h_{x}
$$

Equation (29) corresponds to the set of admissible state which is denoted by $\mathcal{X}_{a d}$ and for the system (22) or (23) can be written as,

$$
\mathcal{X}_{a d}=\left\{x \in \mathbb{R}^{3} \quad \mid \quad H_{x} x \leq h_{x}\right\}
$$

Let's introduce the maximal asymptotic safe set as: 
Definition $7 \mathrm{~A}$ set $\mathcal{C}_{\infty} \subset \mathcal{X}_{a d}$, is said to be the maximal asymptotic safe set for the autonomous system (20) subject to constraints (29), if the evolution of any state belonging to this set stay within the admissible set for all future time and for any disturbance $\omega(k) \in \mathcal{W}$. More formally, the maximal asymptotic safe set can be written as,

$x(k) \in \mathcal{C}_{\infty} \Rightarrow f_{a}(x(k), \omega(k)) \in \mathcal{X}_{a d}, \quad \forall \omega \in \mathcal{W}, k=1,2, \ldots$

The maximal asymptotic safe set $\left(\mathcal{C}_{\infty}\right)$ is conservative since it counts for all possible disturbances $\omega \in \mathcal{W}$. The following algorithm can be used to calculate $\mathcal{C}_{\infty}$.

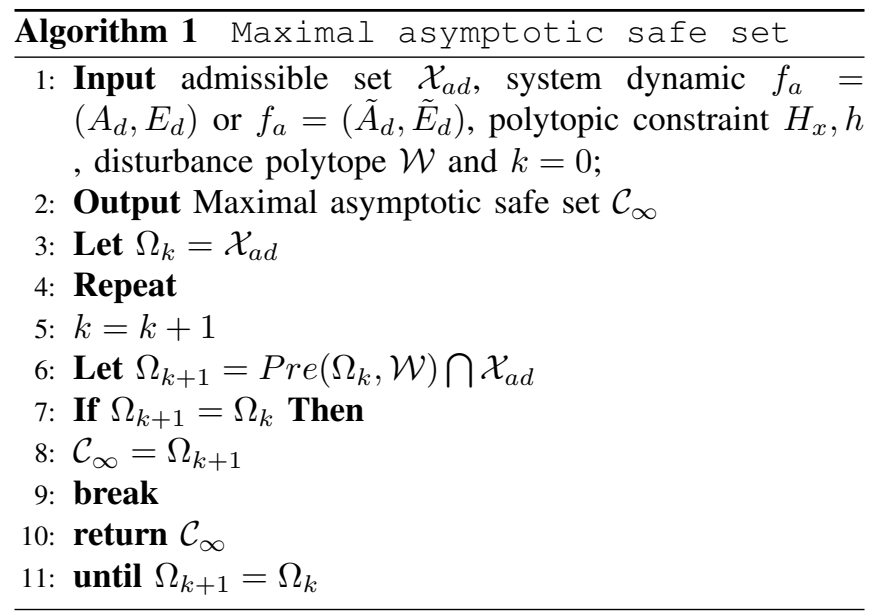

As can be seen from Algorithm (1), computing maximal asymptotic safe set, requires to compute the robust Pre set. For the dynamical system (23), robust Pre set of $\operatorname{Pre}^{f_{a}}(\mathcal{X}, \mathcal{W})$, can be written as,

$$
\operatorname{Pre}(\mathcal{S}, \mathcal{W})=\left\{x \in \mathbb{R}^{3}: H_{x} \tilde{A} x \leq h_{x}-\tilde{E} \omega(k), \forall \omega \in \mathcal{L}(3,2)\right.
$$

The set (32), can be equivalently written as,

$$
\begin{gathered}
\operatorname{Pre}(\mathcal{S}, \mathcal{W})=\left\{x \in \mathbb{R}^{3}: H_{x} \tilde{A} x \leq \tilde{h}, \forall \omega \in \mathcal{W}\right\}, \\
\text { where } \quad \tilde{h}^{i}=\min _{w} h_{x}^{i}-H_{x}^{i} \omega \\
\text { s.t } \quad \omega \in \mathcal{W},
\end{gathered}
$$

where $h_{i}$ is the row-wise minimum of the objective function. The optimization problem (33), can be solved efficiently using linear programming (LP) solvers. The robust Pre set $\operatorname{Pre}^{f_{a}}(\mathcal{X}, \mathcal{W})$ can be also calculated using Pontryagin difference and affine mapping of polyhedra as,

$$
\operatorname{Pr} e^{(\tilde{A}, \tilde{E})}(\mathcal{X}, \mathcal{W})=(\mathcal{X} \ominus \tilde{\mathcal{W}}) \circ \tilde{A},
$$

where $\tilde{\mathcal{W}}=\tilde{E} \circ \mathcal{W}$.

In Fig. 5, the admissible set $\mathcal{X}_{a d}$ and the maximal asymptotic safe set of CACC and ACC controllers for a headway time $h=0.5 s$ are depicted, respectively. The green boxes are the projection of admissible set $\mathcal{X}_{a d}$ on $\left(e_{p}, e_{v}\right)$ where $e_{p, \max }=3 m, e_{p, \min }=-3 m, e_{v, \max }=4 \frac{m}{\mathrm{~s}}$ and $e_{p, \min }=-4 \frac{\mathrm{m}}{\mathrm{s}}$ are the maximum/minimum of constraints on the position and velocity errors, respectively. The red figure represents the maximal asymptotic safe set for a disturbance $\omega \in[-4,-1]$. The figures indicate a slightly larger asymptotic safe set for CACC controller in this case. However, in this work the focus is on the concept of asymptotic safe set rather than comparing the asymptotic safe set of the two controllers, since it requires further investigation. The blue curves show simulations from random initial conditions. As can be seen, for initial conditions outside the red figures, the trajectories evolve outside the admissible set for some disturbances $\omega(k) \in \omega$, while for any initial condition within the $\mathcal{C}_{\infty}$, constraint satisfaction is guaranteed. In Fig. 6, the asymptotic safe set of CACC controller is shown for a shorter headway time $h_{i+1}=0 s$. As can be seen from the figure the maximal asymptotic safe set is smaller for a shorter headway time.

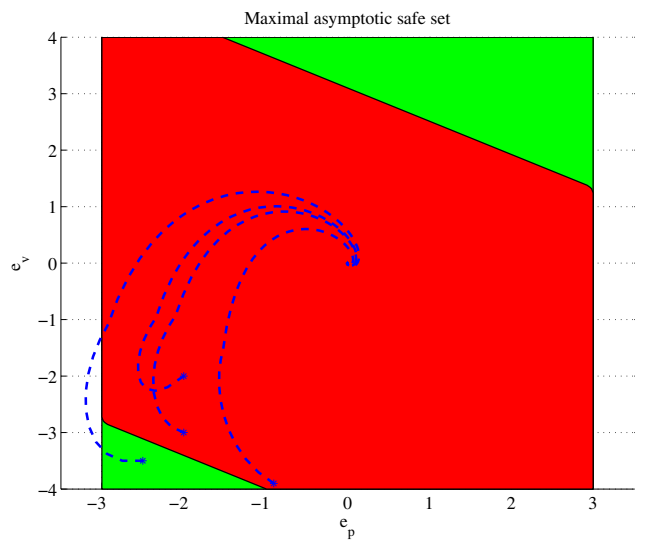

(a) Green box and red figure represent the admissible set and maximal asymptotic safe for CACC controller, respectively

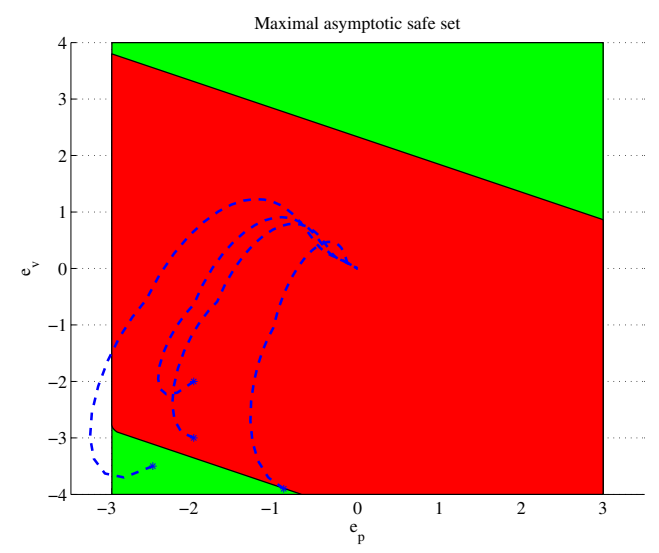

(b) Green box and red figure represent the admissible set and maximal asymptotic safe for ACC controller, respectively

Fig. 5. Blue dashed curves shows the simulation results from random initial points. Stars represent the initial conditions.

\section{RoBUSTNESS ANALYSIS}

The analysis presented in the previous section was done under the assumption of perfect knowledge of the system. However, the results can be extended to a model with parametric uncertainty, i.e., uncertainty in the time constant $\tau_{i+1}$ and static gain $K_{i+1}$. The uncertainty in (2) may arise from uncertainty in the time constant or static gain of actuator. Here, the analysis is limited to the CACC, i.e., closed loop system (23), but the same approach can be applied to ACC (22). Uncertainty in (2), results in uncertainty in matrix $\tilde{A}_{d}$. Assume that the parametric uncertainty in $\tilde{A}_{d}$ 


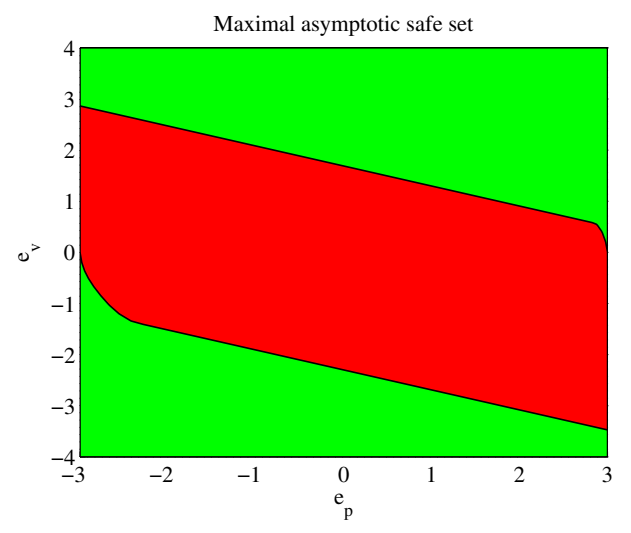

Fig. 6. Admissible set and the maximal asymptotic safe set are shown in green and red, respectively

can be represented by polytopic uncertainty, i.e., $\tilde{A}_{d} \in \tilde{\mathcal{A}}$ $\tilde{\mathcal{A}}=\left\{\tilde{A_{d}} \in \mathbb{R}^{n \times n} \mid \tilde{A_{d}}=\sum_{i=1}^{N_{A}} \lambda_{i} \tilde{A}_{d i}, \quad \sum_{i=1}^{N_{A}} \lambda_{i}=1, \quad \lambda_{i} \geq 0\right\}$,

where $\tilde{A}_{d i}$ are the vertices of the polytope $\tilde{\mathcal{A}}$ and $N_{A}$ is the number of vertices. The Pre operator for the system (23) with parametric uncertainty defined in (36), can be calculated as,

$$
\operatorname{Pr} e^{\left(\tilde{A}\left(\omega_{p}\right), \tilde{E}\right)}(\mathcal{X}, \mathcal{W})=\bigcap_{\tilde{A}\left(\omega_{p}\right) \in \tilde{\mathcal{A}}} \operatorname{Pr} e^{\left(\tilde{A}\left(\omega_{p}\right), \tilde{E}\right)}(\mathcal{X}, \mathcal{W}),
$$

where $w_{p} \in \mathcal{W}_{p}$ represents the parametric uncertainty in the system matrix. Computing Pre in (37), requires infinite number of polytope intersection. However, it can be shown that equation (37), may be computed as finite number of intersections, i.e.,

$$
\operatorname{Pre} e^{\left(\tilde{A}\left(\omega_{p}\right), \tilde{E}\right)}(\mathcal{X}, \mathcal{W})=\bigcap_{i=1}^{N_{A}} \operatorname{Pr} e^{\left(\tilde{A_{d i}}, \tilde{E}\right)}(\mathcal{X}, \mathcal{W})
$$

Proof of (38) is given in [16]. In this study we consider the effect of uncertain time constant $\tau_{i+1}$ in (2). We assume that the time constant $\tau_{i+1} \in[0.3,0.8]$ and the headway time $h_{i+1}=0.5$. The maximal asymptotic safe set for the nominal system (3) with $\tau_{i+1}=0.5$ and the asymptote safe set for the system with uncertain time constant is depicted in Fig. 7. As can be seen from Fig. 7, maximal asymptotic set is shrinking when the parametric uncertainty is taken to account, which seems very reasonable.

\section{CONCLUSION}

In this paper a tool based on reachability analysis and invariant set theory is introduced for safety analysis of a platoon of vehicles equipped with CACC and/or ACC. It is shown that using forward reachability analysis, the reachable set of ACC and CACC controller can be computed. This analysis can be used to design the minimum safety distance for such controller where the closed loop dynamics is subject to disturbance. The results not only indicate that a shorter inter-vehicle distance can be achieved using CACC but also provide a quantitative measure for such comparison. Furthermore, the concept of asymptotic safe set is introduced. This type of analysis provide a tool for analyzing safety, performance, effect of uncertainty and so on.

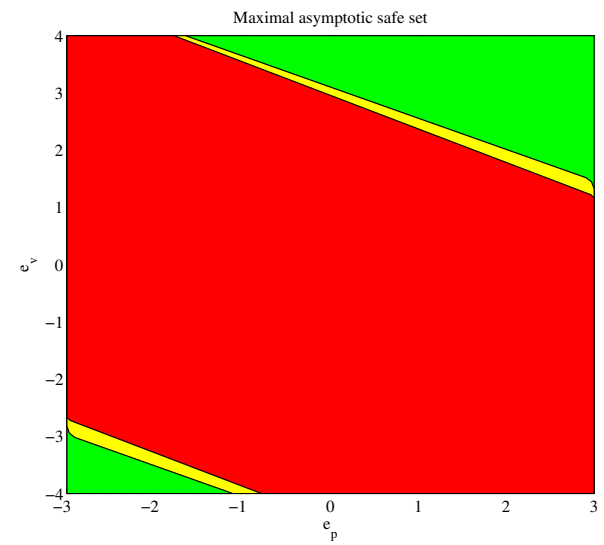

Fig. 7. Green box is the admissible set. The maximal asymptotic safe set for the nominal case and for the system with parametric uncertainty are plotted on top of each other, in yellow and red, respectively

\section{REFERENCES}

[1] S. E. Shladover, Effects of Cooperative Adaptive Cruise Control on Traffic Flow: Testing Drivers' Choices of Following Distance, California PATH Research Report, 2009.

[2] "California Partners for Advanced Transportation TecHnology (PATH)," http://www.path.berkeley.edu.

[3] R. Olfati-Saber, J. A. Fax, and R. M. Murray, "Consensus and cooperation in networked multi-agent systems," Proceedings of the IEEE, vol. 95, no. 1, pp. 215-233, January 2007.

[4] P. Seiler, A. Pant, and K. Hedrick, "Disturbance propagation in vehicle strings," IEEE TRANSACTIONS ON AUTOMATIC CONTROL, vol. 49, no. 10 , pp. 1835-1841, 2004.

[5] D. Swaroop and K. Hedrick, "String stability of interconnected sysyems," IEEE TRANSACTIONS ON AUTOMATIC CONTROL, vol. 41, pp. 349-357, 1996.

[6] R. Rajamani, "Semi-autonomous adaptive cruise control systems," in American Control Conference, Minnesotaa, USA, 1999.

[7] G. Arnaout and S. Bowling, "Towards reducing traffic congestion using cooperative adaptive cruise control on a freeway with a ramp," Industrial Engineering and Management, pp. 699-717, Sep. 2011.

[8] R. Kianfar, P. Falcone, and J. Fredriksson, "A Receding Horizon Approach to String Stable Cooperative Adaptive Cruise Control," in 14th IEEE Conference on Intelligent Transportation Systems, Washington, USA, Oct 2011.

[9] G. Naus, R. P. . Vugts, J. Ploeg, J. de Molengraft, and M. Steinbuch, "String-stable CACC design and experimental validation : A frequency-domain approach," IEEE Transaction on vehicular technology, vol. 59, no. 9, pp. 4268-4279, 2010.

[10] S. Klinge and R. Middleton, "Time Headway Requirement for String Stability of Homogeneous Linear Unidirectionally Connected Systems," in 48th IEEE Conference on Decision and Control, Shanghai, P.R. China, Dec 2009.

[11] R. Rajamani, Vehicle Dynamics and Control. Springer, 2005.

[12] J. P. Maschuw, G. C. Keßler, and D. Abel, "LMI-based Control of Vehicle Platoons for Robust Longitudinal Guidance," in 17th IFAC World Congress, Seoul, Korea, July 2008.

[13] I. Kolmanovsky and E. Gilbert, "Maximal Output Addmissible Sets for Discrete-Time Systems with Disturbance Input," in American Control Conference, Seattle, USA, June 1995.

[14] S. V. Raković, E. C. Kerrigan, K. I. Kouramas, and D. Q. Mayne, "Invariant Approximation of the Minimal Robustly Positively Invariant Set," IEEE Transaction on Automatic Control, vol. 50, no. 3, pp. 406410, 2005.

[15] M. Kvasnica, P. Grieder, and M. Baotić, "Multi-Parametric Toolbox (MPT)," 2004. [Online]. Available: http://control.ee.ethz.ch/ mpt/

[16] H. Lin, X. D. Koutsoukos, and P. J. Antsaklis, "Hierarchical Control for a Class of Uncertain Piecewise Linear Hybrid Dynamical Systems," in 15th IFAC world congress, Barcelona, Spain, 2002. 